\title{
On the growth and form of the gut
}

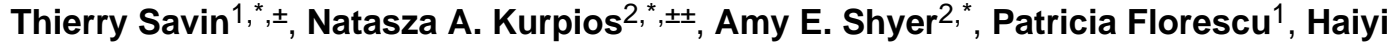 \\ Liang $^{1, \pm \pm \pm}$, L. Mahadevan ${ }^{1,3,4,5,6, \# \text {, and Clifford J. Tabin }}{ }^{2}$ \\ ${ }^{1}$ Engineering and Applied Sciences, Harvard University, Cambridge, MA 02138 \\ ${ }^{2}$ Department of Genetics, Harvard Medical School, Boston, MA 02115 \\ ${ }^{3}$ Organismic and Evolutionary Biology, Harvard University, Cambridge, MA 02138 \\ ${ }^{4}$ Physics, Harvard University, Cambridge, MA 02138 \\ ${ }^{5}$ Wyss Institute for Biologically Inspired Engineering, Harvard University, Cambridge, MA 02138 \\ ${ }^{6}$ Kavli Institute for Nanobio Science and Technology, Harvard University, Cambridge, MA 02138
}

\section{Summary}

The developing vertebrate gut tube forms a reproducible looped pattern as it grows into the body cavity. Here we use developmental experiments to eliminate alternative models and show that gut looping morphogenesis is driven by the homogeneous and isotropic forces that arise from the relative growth between the gut tube and the anchoring dorsal mesenteric sheet, tissues that grow at different rates. A simple physical mimic, using a differentially strained composite of a pliable rubber tube and a soft latex sheet is consistent with this mechanism and produces similar patterns. We devise a mathematical theory and a computational model for the number, size and shape of intestinal loops based solely on the measurable geometry, elasticity and relative growth of the tissues. The predictions of our theory are quantitatively consistent with observations of intestinal loops at different stages of development in the chick embryo. Our model also accounts for the qualitative and quantitative variation in the distinct gut looping patterns seen in a variety of species including the quail, finch and mouse illuminating how the simple macroscopic mechanics of differential growth drives the morphology of the developing gut.

Understanding morphogenesis, the origin of shape in anatomical structures, organs and organisms, has been a central goal of developmental biology since its origins. Historically, the subject focused on the morphology and dynamics of embryonic growth ${ }^{1}$ with many analogies to observable physical phenomena. This metaphoric approach to biological shape is epitomized in D'Arcy Thompson's On Growth and Form², with its emphasis on a mathematical and physical approach to the subject, emphasizing the role of differential growth in determining form. However, with the modern revolution in molecular biology, the field focused on a framework built around gene regulation, signaling molecules and transcription factors. This had led to much insight into the logic of the developmental networks controlling processes as diverse as the patterning of the limb skeleton ${ }^{3}$ and the

\footnotetext{
\#Correspondence should be addressed to: L.Mahadevan, 1m@seas.harvard.edu, Phone: 617-496-9599, Fax: 617-495-9837.

*These authors contributed equally to this work.

${ }^{ \pm}$Current Address: Department of Materials, Polymer Physics, ETH Zürich, 8093 Zürich, Switzerland

${ }^{ \pm \pm}$Current Address: Department of Molecular Medicine, Cornell University, Ithaca, NY 14853

${ }^{ \pm \pm \pm}$Current Address: Department of Modern Mechanics, USTC, Hefei, China
}

Author contributions: CT, NK, LM conceived of project and designed research with additional contributions from TS, AS; TS, NK, AS, LM performed research (TS - biophysical and computational experiments, data analysis, NK - biological experiments, AS biological and biophysical experiments, LM - physical mechanism, physical/mathematical model, scaling theory); PF, HL contributed tools (PF - stitched physical model, HL - built computational model); TS, NK, LM, CT wrote the paper. 
branching morphogenesis of the lung ${ }^{4}$. More recently, however, there has been a renewed appreciation for the fact that to understand morphogenesis in three dimensions, one must combine the molecular insights (genes and morphogens) wit the knowledge of physical processes (transport, deformation and flow) generated by growing tissues.

In this context, there has been only recent limited exploration of the role of tissue-scale mechanical forces in organogenesis ${ }^{5-10}$. Such large-scale forces can become important when the shape of an organ is remodeled after its initial structure has been formed. An important example of this hierarchy is the looping morphogenesis of the gut ${ }^{11}$. The midgut forms as a simple linear tube of circular cross-section running down the midline of the embryo, and grows at a faster rate than the surrounding tissue to eventually become significantly longer than the trunk. As the size of the developing mid- and hindgut exceeds the capacity of the embryonic body cavity, a primary loop is forced ventrally into the umbilicus (in mammals) or yolk stalk (in birds). This loop first rotates by $90^{\circ}$ counterclockwise, followed by another $180^{\circ}$ during the subsequent retraction into the body cavity. Eventually, the rostral half of the loop forms the midgut (small intestine) while the caudal half forms the upper half of the hindgut (the ascending colon).

The chirality of this gut rotation is directed by left-right asymmetries in cellular architecture that arise within the dorsal mesentery ${ }^{12-14}$, an initially thick and short structure along the dorsal-ventral through which the tube is attached to the abdominal wall. This leads the mesentery to tilt the gut tube leftward with a resulting counterclockwise corkscrewing of the gut as it herniates ${ }^{12-13}$. However, the gut rotation is insufficient to pack the entire small intestine into the body cavity, and additional loops are formed as the intestine bends and twists even as it elongates. Once the gut attains its final form, which is highly stereotypical in a given species, the loops retract into the body cavity. During further growth of the juvenile, no additional loops are formed ${ }^{12}$ as they are tacked down by fascia, which restrict movement and additional morphogenesis without inhibiting globally uniform growth.

\section{Relative growth between gut and attached mesentery drives looping}

Throughout development, the gut tube remains attached to the body wall along its entire length by the dorsal mesentery, and is fixed at both its rostral and caudal ends to the mouth and anus, respectively, and thus preserves its connectivity and chirality during growth. The resulting number, shape and size of loops are also conserved in any given species, as shown in Fig. 1a at embryonic day 16 (E16) for the chick.

In principle, this regularity of looping could result from either the intrinsic properties of the gut tube and mesentery, or from external spatial packing constraints. However, surgical dissection of the gut and mesentery from the rest of the embryonic tissues shows that all the loops remain intact and identical to their in ovo structure at various stages of development (Fig. 1a), ruling out any role for body-cavity-induced constraints. Another possible mechanism for the reproducible looping is an increased asymmetric proliferation of cells in the gut tube at the location of the bends. To test this we counted the numbers of mitotic cells in the entire midgut section during the formation of the first loop at E5 (Fig. 1b) and later when there were 9 loops (E12) (Fig. 1b). We observed consistently uniform proliferation with no significant differences along the rostro-caudal axis of the gut tube, including at loop formation locations, and in between loops, as well as no observable azimuthal or radial differences in proliferation rates at different cross-sections (Supplementary Fig. S1), consistent with observations that the embryonic gut tube cross-section remains circular along its length.

Since spatial constraints from the body cavity and the gut tube alone cannot explain the reproducible looping, we turned to the dorsal mesentery, the webbed tissue that attaches the 
gut tube to the embryo throughout its length. As looping morphogenesis is initiated, the dorsal mesentery changes from a thick, asymmetric multilayer structure to a thin doubleepithelial sheet with no observable left-right asymmetry (Supplementary Fig. S2). To test whether the dorsal mesentery is integral to the intestinal loops, we separated it from the gut surgically or enzymatically and find that the intestine uncoils into a straight tube, indicating that it was under compression. Simultaneously, the unconstrained dorsal mesentery when freed from the gut tube contracts (Fig. 1c), indicating that this tissue is under tension. Thus the mesentery-gut composite is required to maintain the mature loops in the gut.

To find out if the dorsal mesentery is also required for the formation of the loops, we surgically separated a portion of the dorsal mesentery from the gut in ovo, beginning immediately caudal to the cranial (superior) mesenteric artery (SMA), at day E4, prior to looping. Strikingly, where the mesentery and gut were separated, the intestinal loops fail to form (Fig. 1d) even as normal loops form in locations rostral and caudal to it (green lines, Fig. 1d). Although we were unable to cut the dorsal SMA in ovo during gut loop development, once the loops had matured (E16), surgical dissection of the SMA leaves the loops intact and in fact highlights their periodic structure (Fig. 2c). This rules out any possible requirement for the SMA in directing loop structure, and for the vasculature as well, since secondary vessels develop only after the loops themselves have formed.

Although the gut grows uniformly, to investigate if the mesentery might grow inhomogeneously and thus force the gut to loop at precise locations, analogous to a master puppeteer, we examined the proliferation rate of the mesentery at E5 and at E12. There were no observed differences along the rostro-caudal axis (Fig. 1b), suggesting that the growing mesentery exerts a uniform compression along the length of the gut, countered by an equal and opposite tensile reaction on it from the gut.

Taken together, our observations suggest that uniform differential growth between the gut and the mesentery could be at the origin of loop formation. Since the gut tube is slender, with a length that is much larger than its radius, it responds physically to the differential strain-induced compression from the attached mesentery by bending and looping, while remaining attached to the embryo rostro-caudally. Most importantly, the fact that the gut relaxes to a straight configuration while the mesentery relaxes to an almost flat configuration implies that the tissues behave elastically, a fact that will allow us to quantify the process simply.

\section{A physical model of gut looping}

To investigate the physical origins of this looping pattern, we developed a simple simulacrum of the gut-mesentery composite using a silicone rubber tube and a thin latex sheet (see Supplementary Information). The differential strain induced by relative growth between the gut and the mesentery is simulated by extending the latex sheet (the mesentery) along its length, and stitching it to the wall of the naturally straight, unstretched rubber tube (the gut) along the edge parallel to the direction of membrane stretching (Fig. 2a). Upon removing all external loads from the composite system, we observe the spontaneous formation of loops in the tube very similar in shape to the looping patterns seen in-ovo (Fig. 2b). Varying the differential strain, the thickness of the latex sheet, the radius of the rubber tube and their material properties (Supplementary Information) shows that the wavelength and amplitude of the repeating loops depends only on these measurable parameters.

\section{Scaling laws for loop period, radius and number}

We now quantify the simple physical picture for looping sketched above to derive expressions for the size of a loop characterized by the contour length $\lambda$ and mean radius of 
curvature $R$ of a single period (see Fig. 3a). The geometry of the growing gut is characterized by its inner and outer radii $r_{i}$ and $r_{o}$, much smaller than its evolving length, while that of the mesentery is described by its homogeneous thickness $h$, which is much smaller than its other two dimensions. Since the tube and mesentery relax to nearly straight flat states once surgically separated, we can model the gut as a one-dimensional elastic filament growing relative to a thin two-dimensional elastic sheet (mesentery). As the gut length becomes longer than the perimeter of the mesentery to which it is attached, there is a differential strain $\varepsilon$ that compresses the tube axially while extending the periphery of the sheet. When the growth strain is larger than a critical value $\varepsilon_{*}$, the tube buckles out of the line to take on a wavy shape of characteristic amplitude $A$ and period $\lambda \gg A$. At the onset of buckling, the extensional strain energy of the sheet $U_{m} \sim E_{m} \varepsilon_{*}^{2} h \lambda^{2}$, where $E_{m}$ is the Young's modulus of the mesentery. The bending energy of the tube $U_{t} \sim E_{t} I_{t} \kappa^{2} \lambda$ where $\kappa \sim A / \lambda^{2}$ is the tube curvature, $I_{t} \sim r_{o}^{4}-r_{i}^{4}$ is the moment of inertia of the tube, and $E_{t}$ is the Young's modulus of the gut tube. Using the condition that the in-plane strain in the sheet $\varepsilon_{*} \sim A / \lambda$ and minimizing the sum of the two energies with respect to $\lambda$ then yields a scaling law for the wavelength of the loop

$$
\lambda \sim\left(\frac{E_{t} I_{t}}{E_{m} h}\right)^{1 / 3} .
$$

The above theory is valid only at the onset of looping and cannot predict the amplitude or radius of a loop. Far from the onset of the instability, at a strain $\varepsilon \varepsilon_{o} \gg \varepsilon_{*}$, we use a torque balance argument to determine the finite radius of the loop. To deform the gut into a loop of radius $R$, the elastic torque required is $T_{t} \sim E_{t} I_{t} / R$ and must balance the torque exerted by the membrane with strain $\varepsilon_{o}$ over a width $W$ and length $R, T_{m} \sim E_{m} h W \varepsilon_{o} R$. The width of this strip is the radial distance from the tube over which the peripheral membrane stretching strain is relaxed, and determined by the relation $\varepsilon_{o} \sim W /(R-W)$. Writing $T_{t} \sim T_{m}$ and assuming $\varepsilon_{0}<1$ yields the scaling law

$$
R \sim\left(\frac{E_{t} I_{t}}{E_{m} h \varepsilon_{0}^{2}}\right)^{1 / 3}
$$

\section{Quantitative geometry and biomechanics of chick gut looping}

A comparison of the results of our predictions with quantitative experiments requires the measurement of the geometry of the tissues, their elastic properties and the relative strain mismatch at different stages of chick gut development; we chose three stages E8, E12, and E16 (Fig. 3). The mesentery has a time-varying thickness $h$, which is evaluated from histological cross-section (supplementary Fig. S2), while the inner/outer radii of the gut tube were extracted from DAPI-stained tube cross-sections (Fig. $3 \mathrm{~b}$ and insets). The length of the gut tube $L_{t}$ was measured on the dissected gut. The natural rest length of the periphery of mesentery $L_{m}$ was measured by cutting out thin strips along the junction with the gut and aligning them unstretched with a ruler (Fig. 3c). The bending stiffness of the gut tube and the stretching stiffness of the mesentery was measured using in vitro uniaxial low-rate tensile tests, where the load was generated by a magnet applying a calibrated force on a millimeter size steel ball, attached on one end of a tissue sample which was pinned at the other end. The extension of the sample under load was tracked using videomicroscopy to extract its stress $\sigma$ vs. strain $\varepsilon$ response curve (see insets of Fig. 3d,e and Methods/ Supplementary information). 
For the mesentery, we observe a non-linear response curve with a sharp break at a strain $\varepsilon \leq$ $\varepsilon_{p}$, where $\varepsilon_{p}=L_{l} / L_{m}-1$ is the physiological strain mismatch, typical of the strain-stiffening seen in biological soft tissues ${ }^{15}$. We define an effective modulus $E_{m}$ and strain $\varepsilon_{o}$ by locally

linearizing the response (see Fig. 3d): $E_{m}=\left(\frac{d \sigma}{d \varepsilon}\right)_{\varepsilon=\varepsilon_{p}}$ and $\varepsilon_{0}=\left(\frac{1}{\sigma} \frac{d \sigma}{d \varepsilon}\right)_{\varepsilon=\varepsilon_{p}}^{-1}$, noting that the membrane has negligible stiffness when $0<\varepsilon<\varepsilon_{p}-\varepsilon_{0}$. For the gut, we measured the modulus $E_{l}=\sigma / \varepsilon$ from the linear low-strain response curve ( $\varepsilon<10 \%$, Fig. 3e). In Fig. 3f,g we summarize the variation of $E_{m}, E_{t}$, and $\varepsilon_{o}$ as a function of developmental time.

Measurements of the mesentery stiffness at various locations and in various directions did not exhibit significant differences (Supplementary Fig. S4). This indeed allows us to model the mesentery and the gut as isotropic, homogeneous material.

The measured biophysical parameters allowed us to create a detailed numerical simulation of gut looping. Since the gut and mesentery grow slowly, inertial forces are unimportant, and the composite system is always in mechanical equilibrium. This configuration was calculated as follows: the mesentery was modeled as a discrete elastic membrane consisting of a hexagonal lattice of springs with a discrete energy associated with in-plane stretching/ shearing deformations as well as out-of-plane bending deformations ${ }^{16}$, relative to the rest length of the springs. The gut was modeled as an equivalent membrane strip (that is two elements wide) with a discretized energy associated with bending and stretching deformations, and elastic stiffnesses different from those of the membrane. The geometry, mechanical properties and relative growth of the tissues parameterized by $h, I_{t}, E_{m}, E_{t}, \varepsilon_{0}$ are all experimentally measured at different time points during development. Given these input parameters, energy minimization for different relative growth strains $\varepsilon_{0}$ yields predictions for the looping morphology of the gut (see Methods, Supplementary Information).

In Fig. 4a, we compare the results of our observation at E16 with numerical simulations, and in Figs. 4b,c we compare our quantitative measurements of the wavelength and radius of curvature of the chick gut at the different measured stages of development (see also Supplementary Fig. S8) with those of both the rubber simulacrum and numerical simulations, as a function of the geometry and elastic moduli of the tube and sheet. Over the range of strain $\varepsilon_{0} \in[0,1]$ in the simulation (Supplementary movie S1) and $\varepsilon_{0} \in[0.5,1]$ for the various rubber models, we plot the wavelength $\lambda$ and radius $R$ of the loop in Fig. 4b,c, and find that they follow the relations

$$
\lambda \simeq 36\left(\frac{E_{t} I_{t}}{E_{m} h}\right)^{1 / 3} \text { and } R \simeq 4\left(\frac{E_{t} I_{t}}{E_{m} h \varepsilon_{0}^{2}}\right)^{1 / 3}
$$

in accord with the previously derived simple scaling laws (1)-(2). Table 1 compares the values of these parameters for the chick gut to those predicted by the model (3), and confirms that our model strikingly captures the salient properties of the looping patterns with no adjustable parameters, strongly suggesting that the main features of the chick gut looping pattern are established by the simple balance of forces induced by the relative growth between the gut and the mesentery.

\section{Comparative study of gut looping across species}

To test our theory beyond the development of the chick gut, we took advantage of the distinct gut looping patterns observed in different avian taxa, which have served as criteria for phylogenetic classification, and are thought of as having adaptive significance, independent of bird size. 
We compared the gut looping patterns of the chick with the closely related (but differently sized) quail and that of a songbird, the zebra finch. In Fig. 5a, we see that, as previously described ${ }^{17,18}$ the gut of the chick and quail are organized almost identically but on different scales, while the digestive tracts of songbirds and chickens are markedly different. To make the comparison quantitative, we repeated the morphometric and mechanical measurements (Supplementary Information) and used these to generate predictions from our scaling theory and computational model. In all cases, the predicted values of $\lambda, R$ and $n$ are again in excellent agreement with those observed in embryonic guts of the appropriate species (Fig. $5 b, c$, Table 2). For instance, we find that, although growth strains $\varepsilon_{p}$ are similar between the chick and quail, the quail mesentery has a tension $E_{m} h \varepsilon_{0}$ approximately five times that in the chick mesentery. Qualitatively, this greater elastic force produces a smaller loop, hence inducing more loops per length and thus the same number of loops in the smaller bird. In contrast, most of the geometrical and physical parameters characterizing the developing gut and mesentery in the chick and zebra finch are different and lead to different looping parameters.

Finally, to challenge our theoretical model with a non-avian example, we performed a similar set of measurements throughout the course of gut development in mouse embryos. In agreement with our findings from birds, the geometrical and biophysical properties of the developing gut and dorsal mesentery suffice to accurately predict the stereotypical patterns of the mature intestinal loops in mouse embryos (Fig. 5, Table 2). The latter are notably characterized by softer tissues and higher mismatch strain, producing tightly coiled loops, as seen in Fig. 5a.

\section{Discussion}

The developing intestine is a simple elongated tubular structure that is stereotypically and reproducibly folded into a compact organ through the process of looping morphogenesis. Our study has shown that the associated looping patterns are quantitatively determined by the differential growth between the gut tube and dorsal mesentery and their geometric and elastic properties, both in an organism and across species using a combination of quantitative experiments, computations and scaling arguments. We thus bring a quantitative biomechanical perspective to the mostly metaphoric arguments in On Growth and Form².

The simplicity of the mechanical origin in the diversity in gut looping patterns, long associated with the adaptive significance of the distinct diets and residence times in different animals ${ }^{17}$ also suggests that since it is sufficient to modulate the uniform tissue growth rates, tissue geometry and elasticity of the gut-mesentery system to change these patterns, these are the minimal properties upon which selection has acted to achieve the looping patterns found in nature.

Identification of the relevant cellular parameters influencing gut morphogenesis opens the door to future studies of the genes involved in controlling cell proliferation and matrix formation in space and time, and sets the stage to understand the processes by which biochemical and biophysical events across scales conspire to drive the developmental regulation of growing tissues.

\section{METHODS SUMMARY}

\section{Embryos}

Fertile chick eggs (White Leghorn eggs) were obtained from commercial sources. Fertile zebra finch eggs were provided by the lab of Tim Gardner at Boston University. Fertile Japanese quail eggs were obtained from Strikland Game Bird. All eggs were incubated at 
$37.5^{\circ} \mathrm{C}$ and staged according to Hamburger and Hamilton ${ }^{19}$. Mouse embryos were collected from stage pregnant females (Charles River Laboratories).

\section{Immunohistochemistry and histology}

Small intestines were collected from chick embryos at desired stages and fixed in $4 \%$ paraformaldehyde in PBS and embedded in paraffin wax. Immunohistochemistry and histology was performed on $10 \mu \mathrm{m}$ transverse sections of the gut tube.

\section{In ovo gut surgeries}

The gut tube and the dorsal mesentery were separated in ovo at stage $23-25$ by using a pulled glass needle to cut the connection between the two tissues. Embryos were reincubated until day 12 when they were collected to examine the resulting looping pattern.

\section{Mechanical properties of gut and mesentery tissue}

The force $F(d)$ between a permanent magnet (The Magnet Source) and millimetric steel balls (New England Miniature Ball Corp.), separated by a distance $d$, was calculated from the damped motion of the ball rising in glycerol whit the magnet approached atop. For $2<d<8$ $\mathrm{mm}$ used in the subsequent measurements, the force $F(d)$ ranges from $1 \mu \mathrm{N}$ to $1 \mathrm{mN}$. We then surgically extracted stripes of the mesentery and sections of the gut tube on fresh animal embryos. A steel bead was attached at one end of the sample, by either gluing it on the stripe or sealing it in the tube. With the other end pinned to an agarose gel, the magnet was approached to stretch the sample, while video-tracking both the distance bead-magnet $d$ and the extension $L$ of the sample. We calculated the stress $\sigma=F(d) / A_{O}$ and the strain $\varepsilon=L / L_{O}-1$ with the length $L_{O}$ and cross-section area $A_{O}$ of the sample at rest. All dissections, manipulations and tensile tests occurred in Ringer buffer (Sigma Aldrich), and within hours after the surgery.

\section{METHODS}

Embryos

Fertile chicken eggs (White Leghorn eggs) were obtained from commercial sources. Fertile zebra finch eggs were provided by the lab of Tim Gardner at Boston University. Fertile Japanese quail eggs were obtained from Strikland Game Bird. All eggs were incubated at $37.5^{\circ} \mathrm{C}$ and staged according to Hamburger and Hamilton ${ }^{19}(\mathrm{HH})$. Mouse embryos were collected from stage pregnant females (Charles River Laboratories).

\section{Immunohistochemistry and histology}

Small intestines were collected from chick embryos at desired stages and fixed in $4 \%$ paraformaldehyde in PBS and embedded in paraffin wax, allowing for $10 \mu \mathrm{m}$ transverse sections of the gut tube. Fast green staining was performed as described in ref. 20. Immunohistochemistry was performed with rabbit polyclonal anti-phospho-H3 (1:100) (Millipore) overnight at 4 degrees in PBS containing 3\% goat serum and 0.1\% Triton X-100. Sections were next incubated with Alexa Fluor 594 goat-anti-rabbit secondary antibody (Molecular Probes) (1:300) for 1 hour at room temperature. DAPI (Molecular Probes) was used as a nuclear counter stain and to determine the size of the inner and outer radii of the gut tube.

\section{In ovo gut surgeries}

The gut tube and the dorsal mesentery were separated in ovo at HH stage 23-25 by using a pulled glass needle to cut the connection between the two tissues. Most, but not all, of the connection was ablated as care was taken to avoid puncturing the dorsal aorta which runs 
over the gut tube and dorsal mesentery at this stage. Embryos were re-incubated until E12 when they were collected to examine the resulting looping pattern.

\section{Physical simulacrum using rubber}

We cut a wide strip of elastic rubber sheet (McMaster-Carr) of various thicknesses. The sheet was held stretched in one direction at the desired extension using clamps, and a silicon rubber tubes (NewAge Industries, inc.), was stitched to the sheet using sewing thread (further details, see Supplementary Information).

\section{Calibration of the magnetic force}

The attractive interaction between a permanent disc magnet (commercial grade, axially magnetized, neodymium Nd-Fe-B; The Magnet Source) and high precision ball bearings' steel balls (AISI 440C stainless steel, radii $\mathrm{r}_{\mathrm{b}}=0.122,0.253$ and $0.398 \mathrm{~mm}$; New England Miniature Ball Corp.) was calibrated using a "falling ball viscometer" geometry: immersed in a tube filled with pure glycerol, the magnet is approached atop the ball that consequently rises (we ensured that all materials used to manipulate the beads and the magnet during the measurements, calibration and tensile tests, had no magnetic susceptibility). The force exerted by the magnet is balanced by gravity, drag and inertia. At low Reynolds number, drag force and inertia can be measured from the ball trajectory that is extracted using video tracking (see details in the Supplementary information). We can then calculate the attractive force $F(d)$ between the magnet and the ball as a function of their separation distance $d$. We report our results in Fig. S3 and Table S1 (supplementary information). Notably, for distances $2<d<8 \mathrm{~mm}$ used in the tissue tensile test described thereafter, the force ranges from $1 \mu \mathrm{N}$ to $1 \mathrm{mN}$.

\section{Measurements of tissue mechanical properties}

We surgically dissected fragments of the mesentery and of the gut tube from live embryos. Samples of the mesentery were cut out to leave a well-defined, constant millimeter-width stripe with principal axis either perpendicular to the tube (radial measurement) or parallel to the tube pathway (tangential measurement). For mesentery fragments, the steel beads were glued using synthetic glue (Instant Krazy Glue) at one end of the tissue stripe. The other end was pinned to an agarose gel layer. During the dissection of the sample, we kept sections of the tube or of the superior mesenteric artery to provide convenient handles to attach the bead (see Fig 3d and Fig S4 of the Supplementary Information, where sections of the tube are clearly visible). For gut tube fragments, the steel beads were inserted into the tube and secured by tying the lumen using a hair with an overhand knot. The other end of the tube was held on the agarose gel using a horseshoe pin (Figs. 3e \& S5). All dissections, manipulations and tensile tests were performed in Ringer buffer (Sigma Aldrich), and the measurements occurred within a few hours after the dissection. The magnet was attached to a plastic arm held on a micrometric translation stage, and approached to the sample on the agarose gel (Fig. S4a). The magnet attracts the steel bead and stretches the sample in a controlled fashion (supplementary movie 2 ). The tensile tests were video-recorded to track the extension $L$ of the sample and the distance bead-magnet $d$, and were ran the following way: the sample was first pre-conditionned by stretching it once to an extension ratio greater than 1, after which the magnet was removed to let the sample relax to its rest length $L_{O}$ (see supplementary movie 2), at which stage we visually extracted the rest width $w O$ of mesentery samples. The magnet was then moved back towards the sample in a step piecewise motion. At each step, the sample stretches and we perform the next step approach of the magnet only after a visual equilibrium extension of the sample is reached. We thus effectively measured the static elasticity of the tissue, by mean of the nominal stress $F(d) / A_{O}$ response to a nominal strain $\varepsilon=L / L_{0}-1$; here $A_{O}$ is the cross section of the sample at rest: $A_{0}=W_{O}\left(1+\varepsilon_{p}\right)^{1 / 2} h$ for the mesentery by virtue of material incompressibility, and 
$A_{0}=\pi\left(r_{o}^{2}-r_{i}^{2}\right)$ for the tube. We further verified that this method returns reproducible results, with same-sample variations below sample-to-sample variations that we measured at about $50 \%$. Several stress-strain response curves, corresponding to samples of mesentery and gut tubes extracted from different Chicken E16 embryos are shown in Figs S4c \& S5b to appreciate the level of reproducibility.

\section{Computational model}

The mesentery is modeled as a hexagonal lattice of linear springs with rest length $a_{m}$, whose discrete energy $F_{m}=\frac{\sqrt{3} E_{m} h}{4} \sum_{i j}\left(r_{i j}-a_{m}\right)^{2}+\frac{E_{m} h^{3}}{12 \sqrt{3}} \sum_{\alpha \beta}\left(\mathbf{n}_{\alpha}-\mathbf{n}_{\beta}\right)^{2}$ accounts for in-plane stretching (first term, where $r_{i j}$ is the spring length between nodes $i$ and $j$ ) and out-of-plane bending (second term, where $\mathbf{n}_{\mathrm{a}}$ is the unit normal vector to the triangular facet) such that it tends to the energy of an elastic membrane of thickness $h$ and modulus $E_{m}$ as $a_{m} \rightarrow 0^{16}$. The gut tube is modeled with a similar 2-element width lattice of springs with rest length $a_{t}$. The

discrete energy $F_{t}=\frac{5.6 \sqrt{3} E_{t} I_{t}}{8 r_{o}^{3}} \sum_{i j}\left(r_{i j}-a_{t}\right)^{2}+\frac{E_{t} I_{t}}{2 r_{o} \sqrt{3}} \sum_{\alpha \beta}\left(\mathbf{n}_{\alpha}-\mathbf{n}_{\beta}\right)^{2}$ of this strip contains also in- and out-of-plane deformations terms (first and second term respectively), which are chosen such that the in- and out-of-plane bending stiffness converge both to $E_{t} I_{t}$ for a tube of outer radius $r_{O}$ (see details in the supplementary information). At various time points in the development of the gut (E8, E12, E16), the parameters by $h, I_{t}, E_{m}, E_{t}, \varepsilon_{0}$ are all experimentally measured and input into the energy, with the relative growth $\varepsilon_{0}=a_{m} / a_{t}-1$ imposing the mismatch strain between the membrane and the tube attached to it. Then the energy $F_{m}+F_{t}$ is minimized by a damped molecular dynamics algorithm ${ }^{16}$, to yield the equilibrium configuration of the gut-mesentery composite system.

\section{Supplementary Material}

Refer to Web version on PubMed Central for supplementary material.

\section{Acknowledgments}

We thank Richard Prum for pointing us to the literature on avian intestines, and the Harvard NSF MRSEC, the MacArthur Foundation (LM) and NIH RO1 HD047360 (CT) for support.

\section{REFERENCES}

1. His, W. Anatomie Menschlicher Embryonen. Leipzig, Germany: Verlag von F.C.W. Vogel; 1880.

2. Thompson, DW. On Growth and Form. Cambridge: Cambridge University Press; 1917.

3. Johnson RL, Tabin CJ. Molecular models for vertebrate limb development. Cell. 1997; 90:979-990. [PubMed: 9323126]

4. Metzger RJ, Krasnow MA. Genetic control of branching morphogenesis. Science. 1999; 284:16351639. [PubMed: 10383344]

5. Hufnagel L, et al. On the mechanism of wing size determination in fly development. Proc. Natl. Acad. Sci. U. S. A. 2007; 104:3835-3840. [PubMed: 17360439]

6. Beloussov LV, et al. Mechanical stresses in embryonic tissues: patterns, morphogenetic role, and involvement in regulatory feedback. Int. Rev. Cyt. 1994; 150:1-34.

7. Taber LA. Biomechanics of cardiovascular development. Annu. Rev. Biomed. Eng. 2001; 3:1-25. [PubMed: 11447055]

8. Salazar-Ciudad I, Jernvall J. A computational model of teeth and the developmental origins of morphological variation. Nature. 2010; 464:583-586. [PubMed: 20220757]

9. Hamant, et al. Developmental patterning by mechanical signals in Arabidopsis. Science. 2008; 322:1650-1655. [PubMed: 19074340] 
10. Forgacs, G.; Newman, S. Biological physics of the developing embryo. Cambridge University Press; 2005.

11. Schoenwolf, SG., et al. Larsen's Human Embryology. United Kingdom: Elsevier Health Sciences; 2008.

12. Kurpios NA, et al. The direction of gut looping is established by changes in the extracellular matrix and in cell:cell adhesion. Proc. Natl. Acad. Sci. U. S. A. 2008; 105:8499-8506. [PubMed: 18574143]

13. Davis NM, et al. The chirality of gut rotation derives from left-right asymmetric changes in the architecture of the dorsal mesentery. Dev. Cell. 2008; 15:134-145. [PubMed: 18606147]

14. Hecksher-Sorensen J, et al. The splanchnic mesodermal plate directs spleen and pancreatic laterality, and is regulated by Bapx1/Nkx3.2. Development. 2004; 131:4665-4675. [PubMed: 15329346]

15. Fung, YC. Biomechanics: Mechanical Properties of Living Tissues. New York: Springer-Verlag; 1993.

16. Liang H, Mahadevan L. The shape of a long leaf. Proc. Natl. Acad. Sci. U. S. A. 2009; 106:2204922054. [PubMed: 19966215]

17. Beddard, FE. The Structure and Classification of Birds. London New York [etc.]: Longmans, Green, and co.; 1898.

18. Mitchell CP. On the intestinal tract of birds. Proc. Zool. Soc. London. 1896; 64:136-159.

19. Hamburger H, Hamilton HL. A series of normal stages in the development of the chick embryo. J. Exp. Morphol. 1951; 88:49-92.

20. Kleinman, RE., et al. Walker's Pediatric Gastrointestinal Disease. Ontario: B.C. Decker; 2008. 

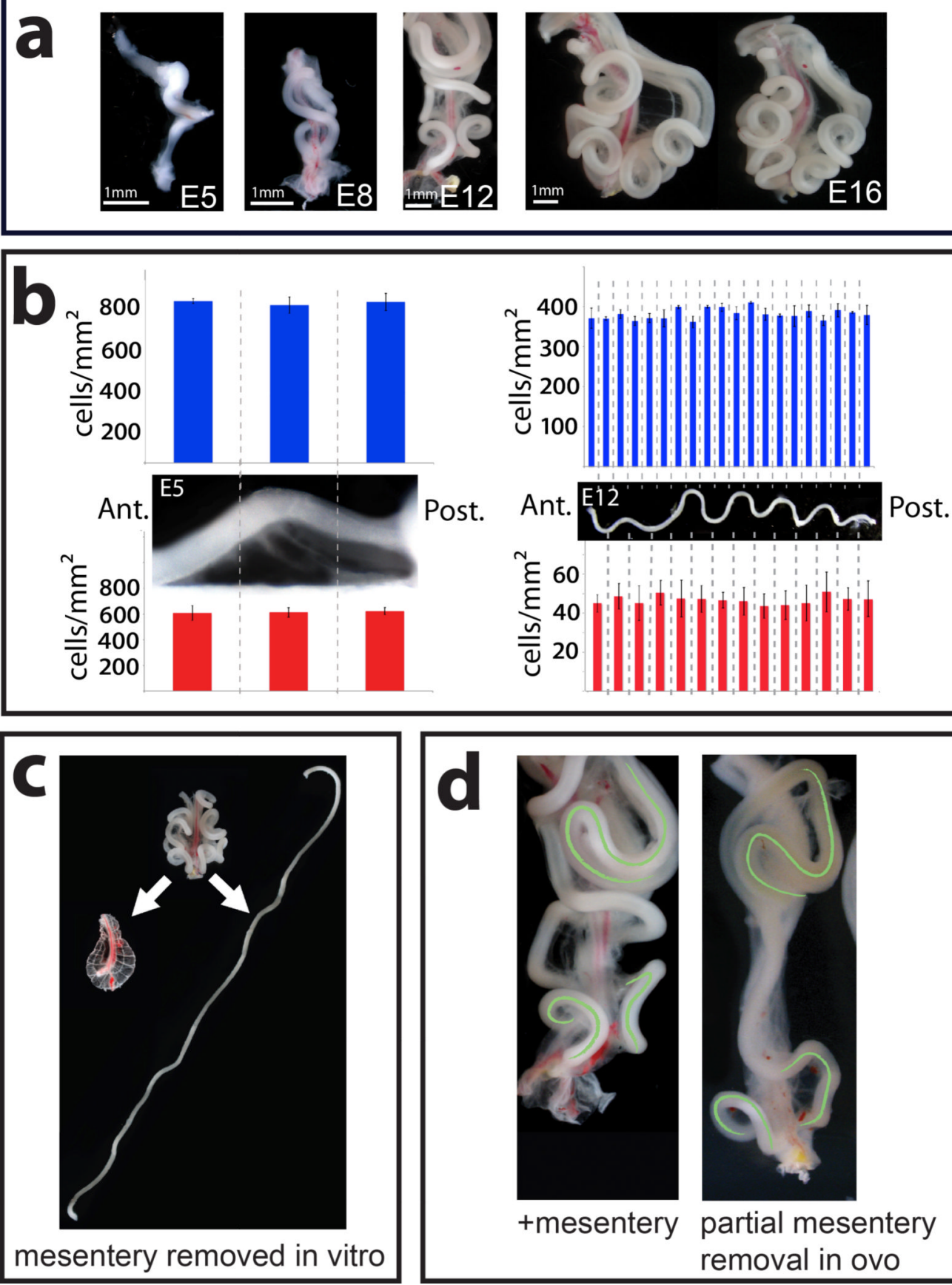

Figure 1. Morphology of loops in the chick gut

a, Chick gut at day 5 (E5), E8, E12, E16 (shows stereotypical pattern). b, Proliferation in the E5 (left) and E12 (right) gut tube (above in blue) and mesentery (below in red). Each blue bar represents the average number of phospho-H3 positive cells per unit surface in 40 (E5) or 50 (E12) $10 \mu \mathrm{m}$ sections. For the mesentery, each red bar represents the average number of phospho-H3 positive cells per unit surface over $610 \mu \mathrm{m}$ sections (E5), or in specific regions demarcated by vasculature along the mesentery (E12). The inset images of the chick guts align the proliferation data with the location of loops (all measurements were done in 3 or more chick samples). c, The gut and mesentery before and after surgical separation at E14 
show that the mesentery shrinks while the gut tube straightens out almost completely. $\mathbf{d}$, The E12 chicken gut under normal development (left) and after in ovo surgical separation of the mesentery at day 4 (right). Note the gut and mesentery repair their attachment, leading to some regions of normal looping (highlighted in green). However a portion of the gut lacks normal loops as a result of disrupting the gut-mesentery interaction over the time these loops would have otherwise developed. 

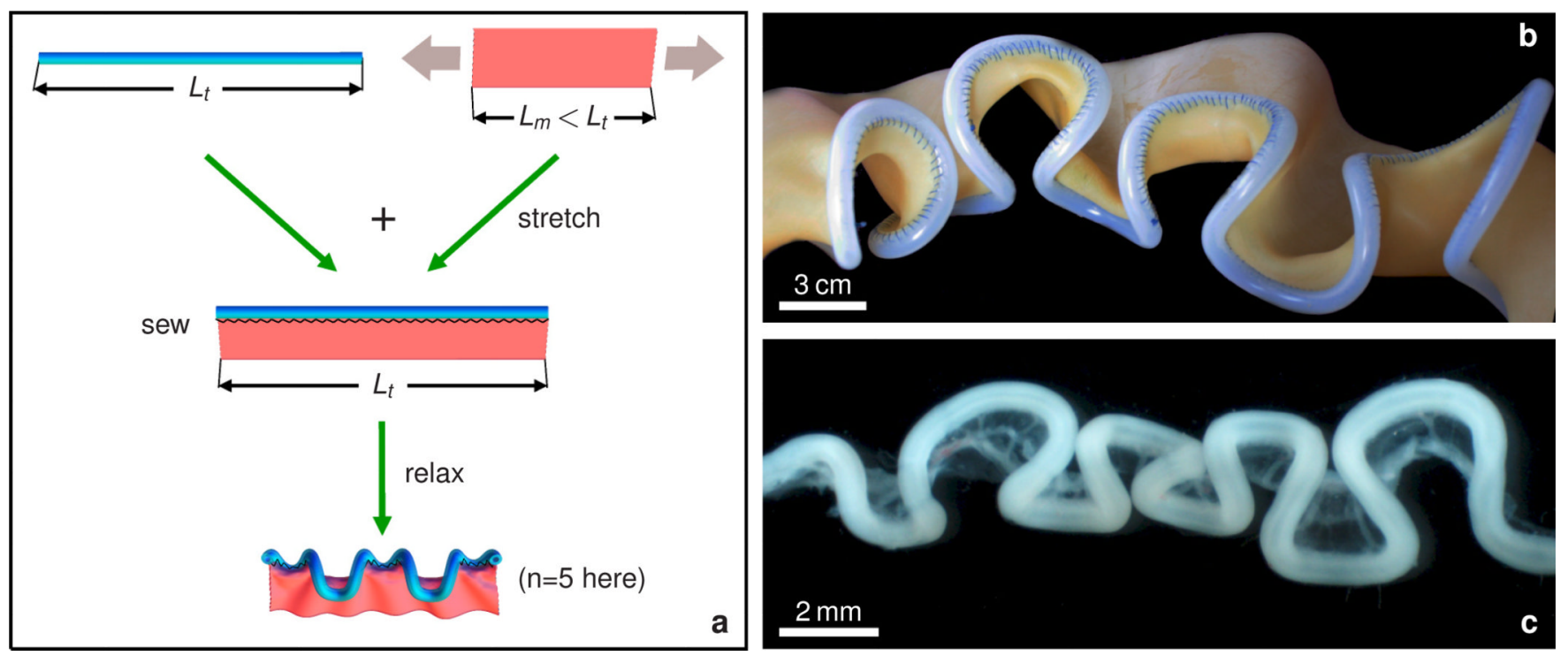

Figure 2. Rubber simulacrum of gut looping morphogenesis

a, To construct the rubber model of looping, a thin rubber sheet (mesentery) is stretched uniformly along its length and then stitched to a straight unstretched rubber tube (gut) along its boundary; the differential strain mimics the differential growth of the two tissues. The system is then allowed to relax, free of any external forces. b, On relaxation, the composite rubber model deforms into a structure very similar to the chick gut (here, the thickness of the sheet is $1.3 \mathrm{~mm}$ and its Young's modulus is $1.3 \mathrm{MPa}$, the radius of the tube is $4.8 \mathrm{~mm}$ its thickness is $2.4 \mathrm{~mm}$, and its Young's modulus is $1.1 \mathrm{MPa}$, see Supplementary Information for details). c, Chick gut at embryonic day E12. The superior mesenteric artery has been cut out (but not the mesentery), allowing the gut to be displayed aligned without altering its loop pattern. 


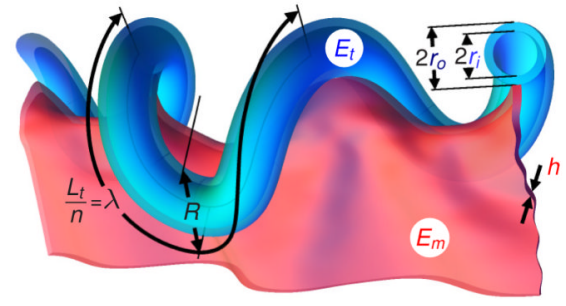

a

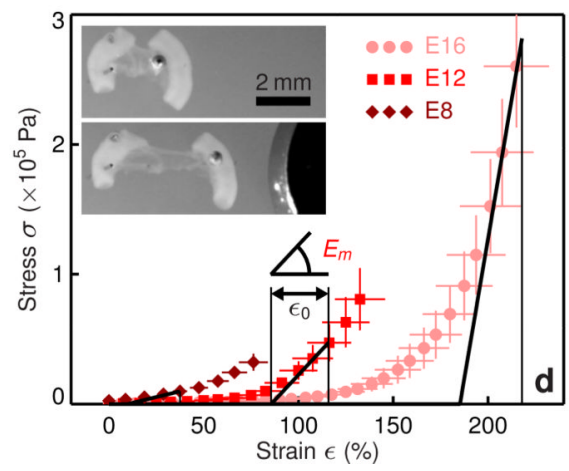

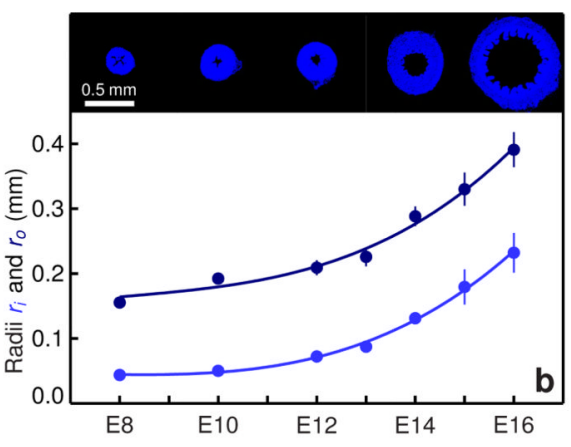
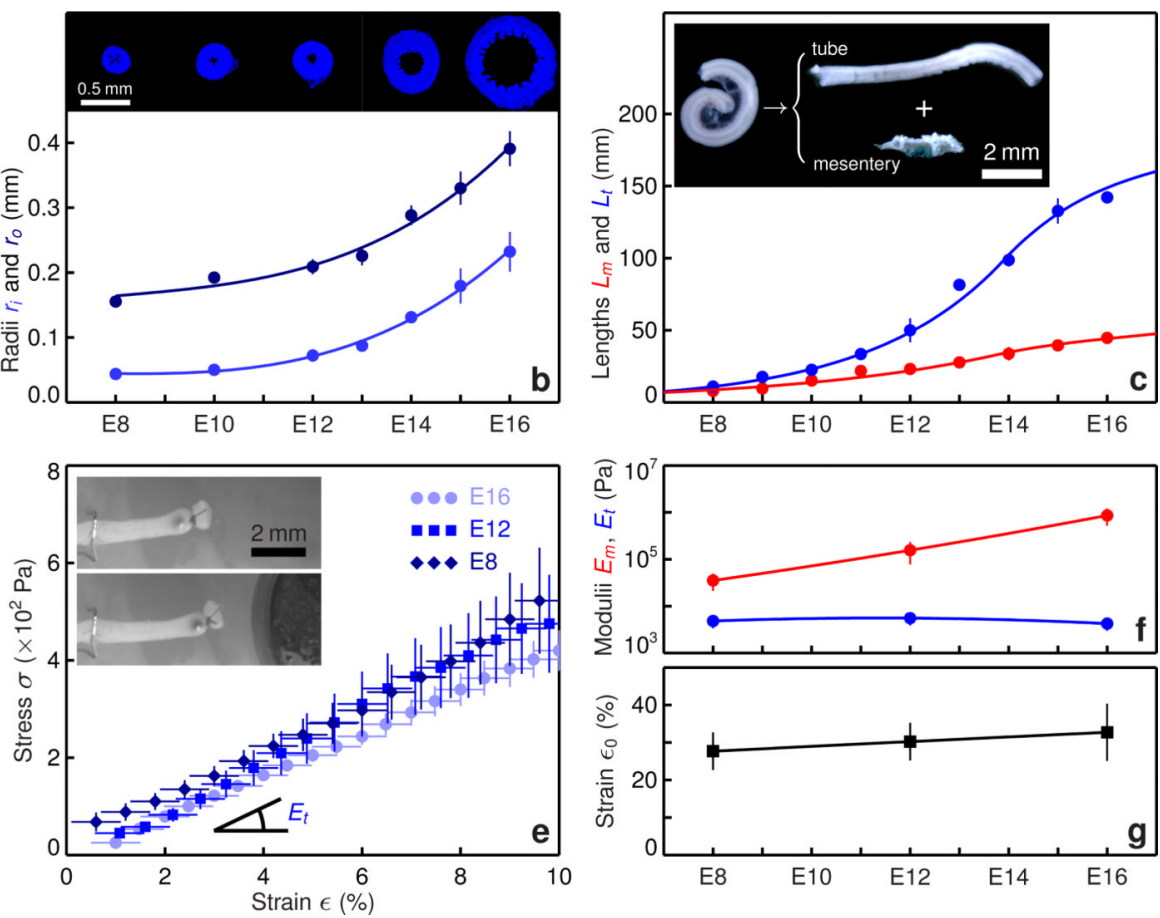

Figure 3. Morphometric and mechanical measurements of chick gut

a, Schematic summarizing the parameters involved in the physical model. b, inner and outer tube diameter. Measurements are extracted from DAPI stained tube cross section shown in insets. c, Tube and mesentery differential growth. Inset shows the length measurement on one isolated loop. d, Stress vs. extension for the mesentery at E8, E12 and E16. The curves are linearized at a characteristic strain corresponding to the physiological strain, as shown by the black lines, to extract the effective Young modulus $E_{m}$ and the effective strain $\varepsilon_{0}$. e, Stress vs. strain curves for the gut tube at E8, E12 and E16. $f$, Mesentery and tube Young's modulii $E_{m}, E_{t}$ as a function of time, E8, E12, E16. $g$, effective differential growth strain $\varepsilon_{0}$ as a function of time, E8, E12, E16. 

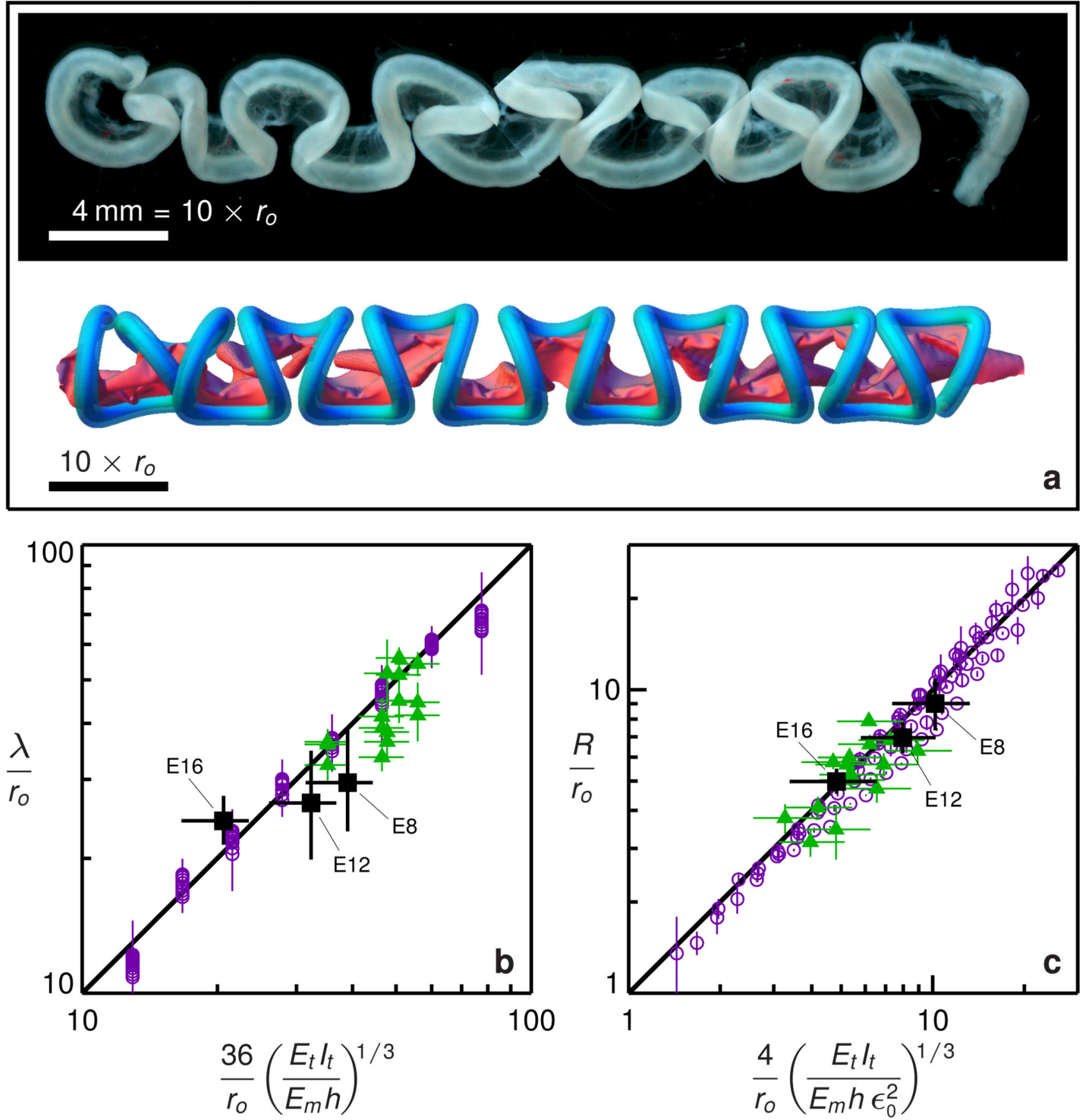

Figure 4. Predictions for loop shape, size and number at 3 stages in chick gut development a, Comparisons of the chicken gut E16 (top) with its simulated counterpart (bottom). b, Scaled loop contour length $\lambda / r_{0}$ plotted vs. eq. (3a) for the chick gut (black squares), the rubber model (green triangles), and numerical simulations (purple circles), are consistent with the scaling law (1). c, Scaled loop radius $R / r_{0}$, plotted vs. eq. (3b), for the chick gut, the rubber model, and numerical simulations, are consistent with the scaling law (2). Symbols are as in b. 

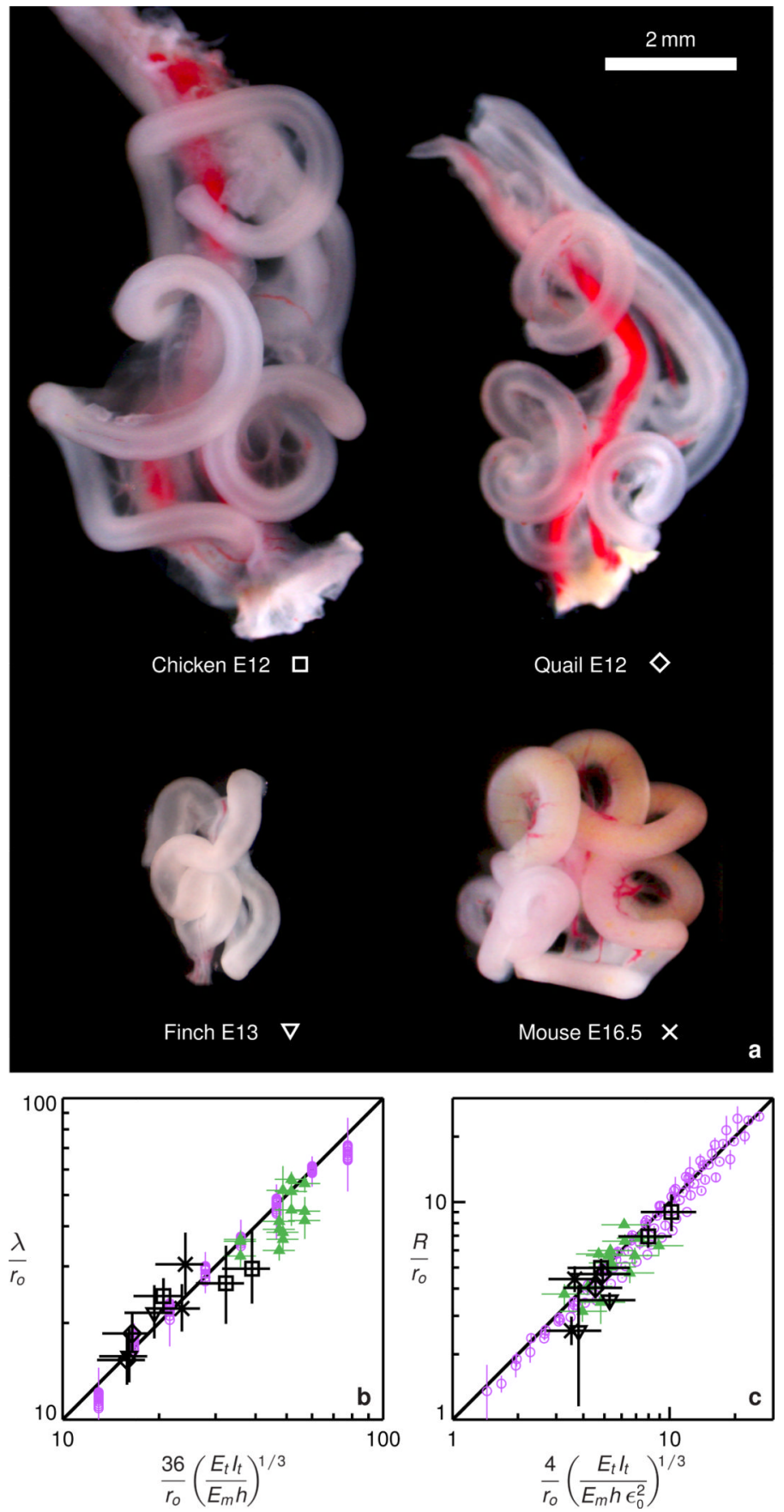

Figure 5. Comparative predictions for looping parameters across species

a, Gut looping patterns in the chick, quail, finch and mouse, to scale show qualitative similarities in the shape of the loops, although the size and number of loops vary substantially. b, Comparison of the scaled contour length $\lambda / r_{0}$ vs. eq. (3a) are consistent with the scaling law (1) for the different species. Black symbols are for the animals shown in (a), other symbols are the same as in fig. 4 b. c, Comparison of the scaled loop radius $R / r_{0}$ vs. eq. (3b) are consistent with the scaling law (2) for the different species. Symbols are as in $b$. In $b$ and c, points for chick E8-12-16, quail E12-15, finch E10-13 and mouse E14.5-16.5 are reported. 


\section{Table 1}

Summary of the observed number of loops, loop wavelength and radius for the chick for different stages of gut development and given geometrical and physical parameters associated with the gut and the mesentery show that the model predictions are quantitatively consistent with observations.

\begin{tabular}{|c|c|c|c|c|}
\hline Chicken & & $n$ & $\lambda(\mathbf{m m})$ & $R(\mathbf{m m})$ \\
\hline \multirow{2}{*}{ E8 } & Experimental observation & $2.4 \pm 0.4$ & $4.6 \pm 1.0$ & $1.4 \pm 0.2$ \\
\hline & Computational model ${ }^{*}$ & $1.8 \pm 0.3$ & $6.1 \pm 1.5$ & $1.6 \pm 0.3$ \\
\hline \multirow{2}{*}{ E12 } & Experimental observation & $9.0 \pm 0.5$ & $5.6 \pm 1.2$ & $1.5 \pm 0.1$ \\
\hline & Computational model ${ }^{\dagger}$ & $7.3 \pm 1.6$ & $6.8 \pm 1.6$ & $1.7 \pm 0.3$ \\
\hline \multirow{2}{*}{ E16 } & Experimental observation & $15.0 \pm 0.5$ & $9.5 \pm 0.5$ & $1.9 \pm 0.1$ \\
\hline & Computational model $\neq$ & $17.5 \pm 2.4$ & $8.1 \pm 1.9$ & $1.9 \pm 0.5$ \\
\hline
\end{tabular}

${ }^{*} L_{t}=11.0 \pm 0.5 \mathrm{~mm}, h=13.0 \pm 1.5 \mu \mathrm{m}, r_{O}=155 \pm 8 \mu \mathrm{m}, r_{i}=44 \pm 5 \mu \mathrm{m}, E_{m}=35 \pm 14 \mathrm{kPa}, E_{t}=4.8 \pm 1.4 \mathrm{kPa}, \varepsilon p=38 \pm 7 \%$, and $\varepsilon O=28 \pm 5 \%$.

${ }^{\dagger} L_{t}=50.0 \pm 8.3 \mathrm{~mm}, h=8.0 \pm 1.5 \mu \mathrm{m}, r_{O}=209 \pm 12 \mu \mathrm{m}, r_{i}=72 \pm 9 \mu \mathrm{m}, E_{m}=156 \pm 78 \mathrm{kPa}, E_{t}=5.6 \pm 1.7 \mathrm{kPa}, \varepsilon p=116 \pm 19 \%$, and $\varepsilon O=30 \pm 5 \%$.

${ }^{*} L_{t}=142.1 \pm 3.3 \mathrm{~mm}, h=7.1 \pm 1.4 \mu \mathrm{m}, r_{O}=391 \pm 27 \mu \mathrm{m}, r_{i}=232 \pm 31 \mu \mathrm{m}, E_{m}=861 \pm 344 \mathrm{kPa}, E_{t}=4.2 \pm 1.3 \mathrm{kPa}, \varepsilon p=218 \pm 15 \%$, and $\varepsilon O=33 \pm 8 \%$. 


\section{Table 2}

Summary of the observed number of loops, loop wavelength and radius for the quail, finch and mouse given the geometrical and physical parameters associated with the gut and the mesentery show that the model predictions are quantitatively consistent with observations.

\begin{tabular}{c|l|c|c|c} 
Species & & $\boldsymbol{n}$ & $\boldsymbol{\lambda}(\mathbf{m m})$ & $\boldsymbol{R}(\mathbf{m m})$ \\
\hline \multirow{2}{*}{ Quail E12 } & Experimental observation & $9.0 \pm 0.7$ & $4.6 \pm 0.4$ & $1.2 \pm 0.1$ \\
\cline { 2 - 5 } & Computational model $^{*}$ & $10.0 \pm 1.3$ & $4.1 \pm 1.0$ & $1.2 \pm 0.3$ \\
\hline \multirow{2}{*}{ Finch E13 } & Experimental observation & $5.5 \pm 0.5$ & $3.6 \pm 0.5$ & $0.6 \pm 0.3$ \\
\cline { 2 - 5 } & Computational model $^{\dagger}$ & $5.3 \pm 0.8$ & $3.7 \pm 0.9$ & $0.9 \pm 0.2$ \\
\hline \multirow{2}{*}{ Mouse E16.5 } & Experimental observation & $6.0 \pm 0.5$ & $6.0 \pm 0.7$ & $0.7 \pm 0.1$ \\
\cline { 2 - 5 } & Computational model & $5.6 \pm 0.8$ & $6.4 \pm 1.5$ & $1.0 \pm 0.1$ \\
\hline
\end{tabular}

${ }^{*} L_{t}=41.3 \pm 0.4 \mathrm{~mm}, h=14.9 \pm 1.6 \mu \mathrm{m}, r_{O}=248 \pm 13 \mu \mathrm{m}, r_{i}=154 \pm 12 \mu \mathrm{m}, E_{m}=515 \pm 206 \mathrm{kPa}, E_{t}=4.4 \pm 1.3 \mathrm{kPa}, \varepsilon p=110 \pm 13 \%$, and $\varepsilon O=23 \pm 5 \%$.

${ }^{\dagger} L_{t}=19.7 \pm 0.8 \mathrm{~mm}, h=6.0 \pm 0.6 \mu \mathrm{m}, r o=227 \pm 14 \mu \mathrm{m}, r_{i}=120 \pm 13 \mu \mathrm{m}, E_{m}=802 \pm 321 \mathrm{kPa}, E_{t}=2.6 \pm 0.8 \mathrm{kPa}, \varepsilon p=110 \pm 11 \%$, and $\varepsilon O=32 \pm 5 \%$.

${ }^{*} L_{t}=35.9 \pm 0.9 \mathrm{~mm}, h=12.3 \pm 1.6 \mu \mathrm{m}, r_{O}=270 \pm 16 \mu \mathrm{m}, r_{i}=178 \pm 14 \mu \mathrm{m}, E_{m}=94 \pm 37 \mathrm{kPa}, E_{t}=1.9 \pm 0.9 \mathrm{kPa}, \varepsilon p=200 \pm 13 \%$, and $\varepsilon O=64 \pm 5 \%$. 